\title{
Partial Wave Spectroscopic Microscopy
}

National Cancer Institute

\section{Source}

National Cancer Institute. Partial Wave Spectroscopic Microscopy. NCI Thesaurus. Code C94595.

A technique that uses focused waves of broadband, low-spatially-coherent light to illuminate a sample, and forms an image by acquiring far field back-scattered photons. A partial spectrum of the back-scattered light intensity is recorded and analyzed. 